\title{
CORRIGENDUM
}

\section{Temporal variation selects for diet-microbe co-metabolic traits in the gut of Gorilla spp}

\author{
Andres Gomez, Jessica M Rothman, Klara Petrzelkova, Carl J Yeoman, Klara Vlckova, \\ Juan D Umaña, Monica Carr, David Modry, Angelique Todd, Manolito Torralba, \\ Karen E Nelson, Rebecca M Stumpf, Brenda A Wilson, Ran Blekhman, Bryan A White \\ and Steven R Leigh
}

The ISME Journal (2016) 10, 532; doi:10.1038/ismej.2015.252

Correction to: The ISME Journal (2016) 10, 514-526; doi:10.1038/ismej.2015.146; published online 28 August 2015

After the publication of this paper, the author noticed an error in the affiliations concerning Klara Petrzelkova and David Modry. The affiliations are as follows:

For Klara Petrzelkova, it is the Institute of Vertebrate Biology, Academy of Sciences of the Czech Republic, Brno, Czech Republic; Biology Centre, Institute of Parasitology, Academy of Sciences of the Czech
Republic, Ceske Budejovice, Czech Republic; and Liberec Zoo, Liberec, Czech Republic.

For David Modry, it is the Department of Pathology and Parasitology, Faculty of Veterinary Medicine, University of Veterinary and Pharmaceutical Sciences Brno, Czech Republic; Biology Centre, Institute of Parasitology, Academy of Sciences of the Czech Republic, Ceske Budejovice, Czech Republic; and CEITEC, Central European Institute for Technology, Brno, Czech Republic.

The authors would like to apologise for any inconvenience this may have caused. 